\title{
Qualidade de vida de adultos e idosos pós adaptação de próteses auditivas
}

\section{Quality of life of adults and elderly people after hearing aids adaptation}

\author{
Adriane Ribeiro Teixeira ${ }^{1}$, Luciane Gomes Almeida ${ }^{2}$, Geraldo Pereira Jotz ${ }^{3}$, Marion Cristine De Barba ${ }^{4}$
}

RESUMO

\begin{abstract}
Objetivo: Verificar se existe melhora na qualidade de vida de adultos e idosos após a adaptação da prótese auditiva e se a variável sexo influencia nos resultados. Métodos: A amostra consistiu de 20 indivíduos, sendo nove mulheres e 11 homens, com idades entre 45 e 81 anos. A coleta de dados foi feita por meio do instrumento WHOQOL-bref, que foi aplicado antes da adaptação da prótese auditiva e após um mês de uso. Resultados: Verificou-se que houve melhora da qualidade de vida, comparando-se os períodos pré e pós-adaptação. Essa melhora foi significante no domínio psicológico, bem como no escore global dos resultados. A variável sexo, no grupo estudado, não influenciou os resultados obtidos. Conclusão: A adaptação da prótese auditiva promoveu a melhora na qualidade de vida dos indivíduos avaliados, independentemente do sexo dos mesmos.
\end{abstract}

Descritores: Qualidade de vida; Auxiliares de audição; Deficiência auditiva; Envelhecimento; Idoso; Adulto; Questionários

\section{INTRODUÇÃO}

O Brasil é um país em desenvolvimento, e, por enquanto, há predominância da população jovem. Entretanto, com a evolução da medicina e com as melhorias na qualidade de vida, a expectativa de vida da população brasileira está aumentando, contribuindo assim para o crescimento da população idosa ${ }^{(1-2)}$. A expectativa é que, em 2020, o país tenha uma população de 32 milhões de pessoas com idade superior a 60 anos $^{(3)}$. Esta é a idade em que os indivíduos passam a ser considerados idosos, em países em desenvolvimento. Este é o ponto de corte cronológico, os efeitos do envelhecimento, no entanto, podem começar a ser sentidos antes desta idade, pela diminuição no desempenho do funcionamento dos mais diversos sistemas do organismo humano.

A deficiência auditiva é uma das dificuldades que mais atingem a população que envelhece e, também, é uma das mais

Trabalho realizado na Universidade Luterana do Brasil - ULBRA - Canoas (RS), Brasil.

(1) Doutora, Fonoaudióloga do Laboratório de Audiologia - LAB - Porto Alegre (RS), Brasil.

(2) Fonoaudióloga clínica, formada pela Universidade Luterana do Brasil ULBRA - Canoas (RS), Brasil.

(3) Doutor, Pós-Doutorado em Otorrinolaringologia na University of Pittsburgh - Pittsburgh (PA), Estados Unidos; Professor Adjunto da disciplina de Otorrinolaringologia do Curso de Medicina da Universidade Luterana do Brasil - ULBRA - Canoas (RS), Brasil e do Departamento de Ciências Morfológicas da Universidade Federal do Rio Grande do Sul - UFRGS - Rio Grande do Sul (RS), Brasil.

(4) Mestre, Fonoaudióloga do Serviço de Alta Complexidade em Saúde Auditiva do Hospital Universitário da Universidade Luterana do Brasil ULBRA - Canoas (RS), Brasil.

Endereço para correspondência: Adriane Ribeiro Teixeira. R. Alberto Rangel, 315/911, Parque Santa Fé, Porto Alegre - RS, CEP 91180-840. E-mail: adriteixeira@yahoo.com.br

Recebido em: 23/10/2007; Aceito em: 19/8/2008 incapacitantes ${ }^{(4)}$. É causadora de vários problemas, tais como: dificuldades de comunicação, isolamento social, depressão e sentimentos negativos, que podem afetar seriamente a qualidade de $\operatorname{vida}^{(5-6)}$. Devido à perda auditiva, há prejuízo nas relações interpessoais do indivíduo, bem como dificuldades para se manter informado pelos meios de comunicação e de usufruí-los como lazer ${ }^{(7-8)}$.

A presbiacusia, ou seja, a deficiência auditiva provocada pelo processo de envelhecimento tem seu início anterior aos 60 anos. Homens e mulheres sofrem com a perda da audição, porém nos homens, o início é precoce (por volta dos 30 anos) e a evolução é mais rápida ${ }^{(9)}$.

Sabendo-se o quanto a perda auditiva interfere na qualidade de vida dos indivíduos, independentemente da idade, adultos e idosos são encaminhados para o processo de reabilitação, que se inicia, em muitos casos, com a seleção e a adaptação de próteses auditivas. Melhorar a capacidade auditiva dos indivíduos significa diminuir a privação sensorial e o handicap, evitando o isolamento e proporcionando-lhes melhora em sua capacidade de interação com o meio em que vivem ${ }^{(10-11)}$.

A audição exerce influência significativa na qualidade de vida, uma vez que o afastamento do meio familiar e social pode originar ou agravar quadros de isolamento ou depressão. O processo de reabilitação auditiva possibilita que os indivíduos retomem a sua vida social, participando de atividades em grupo, melhorando sua auto-estima e bem-estar ${ }^{(12-13)}$.

Definir qualidade de vida, porém, ainda é algo difícil. Muitos conceitos são descritos na literatura especializada, muitas vezes, restritos somente a um aspecto, como a saúde. Em função da amplitude do tema e da multiplicidade de conceituações, optou-se por adotar, neste trabalho, a definição de qualidade de vida da Organização Mundial da Saúde (OMS): “a percepção do indivíduo de sua posição na vida, no contexto da cultura 
e sistema de valores nos quais ele vive e em relação aos seus objetivos, expectativas, padrões e preocupações" (14).

Avaliar a qualidade de vida implica a adoção de múltiplos critérios de natureza biológica, psicológica e sociocultural. São vários os fatores apontados como determinantes ou indicadores, tais como: a longevidade, saúde biológica, satisfação, controle cognitivo, competência social, produtividade, atividade, status social, renda, continuidade de familiares e rede de $\operatorname{amigos}^{(15)}$.

Em função das muitas definições e conceitos sobre o tema, avaliar qualidade de vida também não é uma tarefa simples. Vários instrumentos são encontrados na literatura especializada. Um dos melhores e mais utilizados questionários de avaliação é o desenvolvido pela OMS, pois foi criado dentro de uma escala com perspectiva transcultural, por pesquisadores dos mais variados países, o WHOQOL-100 (14,16-17).

Devido à complexidade e à extensão deste instrumento, a OMS sentiu a necessidade de obter um teste mais resumido para os estudos epidemiológicos mais amplos, porém com características psicométricas satisfatórias. Por isso desenvolveu uma versão abreviada, o WHOQOL-bref ${ }^{(16)}$.

Para manter o mesmo intuito do instrumento WHOQOL-100, a OMS definiu que o caráter abrangente seria preservado. Assim, cada faceta deveria ser representada por uma questão.

OWHOQOL-bref consiste em 26 questões. Duas são questões gerais de qualidade de vida e as demais 24 representam cada uma das facetas derivadas do instrumento de avaliação original. Os instrumentos citados já foram traduzidos e validados para a língua portuguesa ${ }^{(14,16)}$.

A relação entre audição e qualidade de vida já foi abordada por alguns estudos nacionais e internacionais ${ }^{(11-12)}$, mas sempre de forma qualitativa ou por meio de questionários de satisfação com a prótese auditiva, que tratam do tema de forma subjetiva. O uso de instrumentos específicos para avaliação de qualidade de vida no período pré e pós-adaptação de próteses auditivas ainda é incipiente, especialmente na língua portuguesa.

Acreditando-se na interferência negativa da deficiência auditiva na qualidade de vida e na importância da reabilitação auditiva, mais especificamente do uso de próteses auditivas para a adequada interação do indivíduo ao meio e a conseqüente melhora na qualidade de vida, optou-se por realizar este estudo. Assim sendo, os objetivos deste trabalho são: verificar se existe melhora na qualidade de vida de adultos e idosos, após a adaptação da prótese auditiva e se a variável sexo influencia nos resultados obtidos.

\section{MÉTODOS}

A amostra foi composta de 20 indivíduos adultos e idosos, de ambos os sexos, com idades variando entre 45 e 90 anos, oriundos de Porto Alegre, da Região Metropolitana e do interior do Estado do Rio Grande do Sul. Os critérios de inclusão foram os seguintes:

- participar das duas fases do estudo;

- ter idade igual ou superior a 45 anos, na data de realização da primeira avaliação;

- assinatura do Termo de Consentimento Livre Esclarecido (TCLE);

- $\quad$ ser portador de perda auditiva neurossensorial;
- ser candidato ao uso de prótese auditiva e iniciar o uso, pela primeira vez, no Serviço de Otorrinolaringologia e Cirurgia de Cabeça e Pescoço (Divisão de Saúde Auditiva) do Hospital Universitário da Universidade Luterana do Brasil (ULBRA);

- ter capacidade de compreensão para responder ao questionário WHOQOL-Bref;

- ser usuário de prótese auditiva pela primeira vez;

- comparecer aos retornos agendados pelo serviço.

No momento em que o indivíduo comparecia para a avaliação audiológica e era confirmada a presença de perda auditiva e a necessidade do uso de prótese auditiva, era feito o primeiro contato, explicando-se o objetivo do estudo, e o convite para dele participar.

Nos casos de aceite, foi preenchido e assinado o termo de consentimento livre e esclarecido (TCLE) e um questionário elaborado especialmente para este estudo, visando à obtenção de dados sobre os indivíduos incluídos na pesquisa. Foram questionados: idade, estado civil, escolaridade, endereço, telefone, doenças, medicações.

A seguir, foi aplicado o questionário WHOQOL-bref, para a avaliação da qualidade de vida. O WHOQOL-bref é um instrumento de auto-avaliação, por isso, auto-explicativo. Foi enfatizado aos participantes que o questionário deveria ser respondido com base apenas nas duas últimas semanas de sua vida e que deveria ser respondido em apenas um encontro, de forma auto-aplicável. Se o entrevistado não entendia o significado de alguma pergunta, o entrevistador relia a questão de forma lenta, sem utilizar sinônimos ou algum tipo de explicação em outras palavras. Quando o entrevistado não tinha condições de ler o questionário em função de suas condições de saúde ou de alfabetização, o instrumento era lido pelo entrevistador ${ }^{(13)}$.

$\mathrm{Na}$ segunda fase do estudo, realizada quando o indivíduo retornava ao serviço, já utilizando a prótese auditiva, o WHOQOL-bref foi aplicado novamente, seguindo-se as mesmas explicações. O intervalo foi de um mês para todos os indivíduos.

A análise estatística foi executada no software Statistical Package for Social Science (SPSS) 10.0 for Windows ${ }^{\circledR}$. Todos os testes foram realizados na forma bi-caudal, admitindo-se como estatisticamente significantes os valores de p menores ou iguais a 0,05 .

A análise descritiva das variáveis quantitativas foi realizada por meio da observação do cálculo de médias e desvio-padrão. Para as variáveis qualitativas, foi calculada a freqüência absoluta.

A comparação entre o WHOQOL-bref nos momentos pré e pós-protetização foi realizada por meio do Teste t para amostras emparelhadas.

Esta pesquisa foi aprovada pelo Comitê de Ética em Pesquisa da Universidade Luterana do Brasil (protocolo 323H-2006).

\section{RESULTADOS}

A amostra foi composta por 20 indivíduos adultos e idosos, de ambos os sexos (Tabela 1), com idades variando entre 45 e 81 anos. A média de idade foi de 65,30 $( \pm 10,16$ anos $)$. 
A maioria dos sujeitos da amostra era casada (65\%), tendo o primeiro grau como escolaridade máxima (70\%). Todos foram adaptados com prótese auditiva bilateralmente.

Tabela 1. Distribuição dos componentes da amostra segundo o sexo

\begin{tabular}{lcc}
\hline Sexo & N & $\%$ \\
\hline Masculino & 11 & 55 \\
Feminino & 9 & 45 \\
\hline Total & 20 & 100 \\
\hline
\end{tabular}

Legenda: $\mathrm{N}=$ número de sujeitos; $\%$ = porcentagem

Com relação ao grau de perda auditiva (Tabelas 2 e 3), a maioria das orelhas apresentou perda auditiva de grau moderado (60\%). Para a verificação do grau de deficiência auditiva utilizou-se a classificação da Organização Mundial da Saúde ${ }^{(18)}$.

Tabela 2. Grau de deficiência auditiva da OD apresentados pelos componentes da amostra

\begin{tabular}{lcc}
\hline Grau de deficiência auditiva & $\mathrm{N}$ & $\%$ \\
\hline Leve & 1 & 5 \\
Moderada & 12 & 60 \\
Severa & 7 & 35 \\
\hline Total & 20 & 100 \\
\hline
\end{tabular}

Legenda: $\mathrm{N}$ = número de sujeitos; \% = porcentagem

Tabela 3. Grau de deficiência auditiva da OE apresentados pelos componentes da amostra

\begin{tabular}{lcc}
\hline Grau de deficiência auditiva & $\mathrm{N}$ & $\%$ \\
\hline Leve & 1 & 5 \\
Moderada & 12 & 60 \\
Severa & 7 & 35 \\
\hline Total & 20 & 100 \\
\hline
\end{tabular}

Legenda: $\mathrm{N}$ = Número de sujeitos; \% = porcentagem

Os resultados referentes à qualidade de vida pré e pósprotetização encontram-se nas Tabelas 4, 5, e 6.

A Tabela 4 representa os resultados da avaliação da qualidade de vida pré e pós-protetização para o grupo avaliado (20 sujeitos). Os resultados para o grupo do sexo feminino estão representados na Tabela 5 e os resultados para o grupo do sexo masculino na Tabela 6 .

Tabela 4. Comparação dos domínios do WHOQOL-Bref, nos momentos pré e pós-protetização

\begin{tabular}{lccc}
\hline Domínio & Pré-protetização & Pós-protetização & Valor $p$ \\
\hline Físico & $51,96 \pm 14,31$ & $54,64 \pm 11,71$ & 0,287 \\
Psicológico & $57,08 \pm 14,18$ & $64,79 \pm 9,22$ & $<0,0001^{*}$ \\
Social & $62,50 \pm 15,65$ & $64,17 \pm 17,75$ & 0,635 \\
Meio ambiente & $46,41 \pm 13,31$ & $50,16 \pm 11,58$ & 0,057 \\
\hline Total & $83,60 \pm 13,43$ & $89,05 \pm 9,55$ & $<0,0001^{*}$ \\
\hline
\end{tabular}

Número de sujeitos $=20$, média \pm desvio padrão
Tabela 5. Resultados do WHOQOL-bref no sexo feminino no pré e pós-protetização

\begin{tabular}{lccc}
\hline Domínio & Pré-protetização & Pós-protetização & Valor $p$ \\
\hline Físico & $46,43 \pm 15,57$ & $49,60 \pm 8,83$ & 0,531 \\
Psicológico & $54,17 \pm 14,43$ & $62,04 \pm 9,87$ & $0,002^{*}$ \\
Social & $61,11 \pm 20,41$ & $65,74 \pm 16,90$ & 0,416 \\
Meio ambiente & $45,49 \pm 15,87$ & $47,22 \pm 14,01$ & 0,499 \\
\hline Total & $80,22 \pm 16,57$ & $86,11 \pm 10,37$ & $0,046^{*}$ \\
\hline
\end{tabular}

Número de sujeitos $=9$, média \pm desvio padrão

Tabela 6. Resultados do WHOQOL-bref no sexo masculino no pré e pós-protetização

\begin{tabular}{lccc}
\hline Domínio & Pré-protetização & Pós-protetização & Valor $p$ \\
\hline Físico & $56,49 \pm 12,04$ & $58,77 \pm 12,51$ & 0,341 \\
Psicológico & $59,47 \pm 14,20$ & $67,05 \pm 8,43$ & $0,006^{*}$ \\
Social & $63,64 \pm 11,35$ & $62,88 \pm 19,14$ & 0,871 \\
Meio ambiente & $47,16 \pm 11,56$ & $52,56 \pm 9,14$ & 0,074 \\
\hline Total & $86,36 \pm 10,22$ & $91,45 \pm 8,55$ & $0,007^{*}$ \\
\hline
\end{tabular}

Número de sujeitos $=11$, média \pm desvio padrão

Por meio de Teste t para amostras emparelhadas, verificouse que houve diferença estatisticamente significante entre os resultados pré e pós-protetização no domínio psicológico e também nos escores totais, considerando-se o grupo como um todo e também os sujeitos de cada um dos sexos.

\section{DISCUSSÃO}

A presença de deficiência auditiva bilateral, de grau moderado é muito comum na faixa etária dos indivíduos que compuseram a amostra ${ }^{(19-20)}$. Nos sujeitos tanto dos gêneros masculino como feminino, constatou-se que, em ambas as orelhas, a perda de grau moderado foi a mais prevalente. A literatura especializada evidencia que a audição é um dos primeiros sentidos a apresentar perda com o passar do tempo, sendo que os indivíduos começam a perceber, a partir dos 30 anos, algum grau de envelhecimento da audição, que aumenta progressivamente, com o avanço da idade (7,9,21-22). $^{\text {. }}$

Os resultados apresentados evidenciam que a qualidade de vida dos indivíduos que compuseram a amostra melhorou após a adaptação da prótese auditiva, comprovando a importância da audição. No domínio psicológico, bem como na análise total, constataram-se diferenças estatisticamente significantes. Nos domínios físico, meio ambiente e social diferenças significativas não foram obtidas, mas foi constatada melhora nos escores, o que mostra uma melhora global, quando comparados os dados pré e pós-adaptação.

As diferenças eram esperadas pelos pesquisadores, uma vez que se acreditava na melhora da qualidade de vida com o uso de prótese auditiva, especialmente pela melhora na capacidade de comunicação ${ }^{(12-13,23-24)}$, possibilitando o retorno dos indivíduos à vida familiar e social. Considerando-se que a comunicação é imprescindível para a vida em grupo e que a deficiência auditiva faz com que o processo comunicativo seja rompido, no momento em que a reabilitação é iniciada, ocorre um retorno do indivíduo ao mundo sonoro. Isto faz com que 
ele e a família tenham condições de desempenhar atividades sociais, que envolvam os processos comunicativos.

Analisando-se os resultados obtidos na pré e na pós-protetização, por domínio, as respostas estavam parcialmente de acordo com a hipótese inicial dos pesquisadores. Acreditava-se que a melhora na qualidade de vida dar-se-ia especificamente nos domínios social e psicológico, pela melhora na capacidade de comunicação, porém isto não foi constatado.

O domínio psicológico teve melhora significativa no momento da pós- protetização, confirmando a importância do aspecto auditivo para a qualidade de vida e saúde geral do indivíduo $^{(13)}$. Contrariando o esperado, porém, não houve melhora significativa no domínio social. Isto pode ser explicado pela ausência de mudança nos hábitos sociais e no estilo de vida dos indivíduos avaliados. Acreditava-se que os sujeitos participariam mais ativamente dos grupos, tendo melhor inserção na sociedade e evitando assim o isolamento social ${ }^{(10)}$. Talvez se houvesse re-aplicação do protocolo em um tempo superior a um mês, os resultados fossem diferentes ${ }^{(12)}$. Outro fator a ser considerado é que talvez o isolamento, atribuído somente à deficiência auditiva, seja provocado por outras causas, tais como: a residência unifamiliar (idoso residindo sozinho) ou o afastamento entre o idoso e a família, provocado não somente pela questão auditiva, mas por outros fatores ambientais, emocionais e sociais ${ }^{(7,10,24-25)}$. Outro aspecto que pode ter influenciado este resultado é que, dentro do primeiro mês de adaptação, os sujeitos são orientados a utilizar a prótese auditiva em ambientes calmos, evitando locais onde o ruído seja excessivo, o que pode levar os novos usuários a evitarem os ambientes sociais externos ao lar. Esta orientação é dada porque, como os sujeitos pertencem a um grupo que recebe próteses auditivas pelo Programa de Alta Complexidade em Saúde Auditiva, do Ministério da Saúde, seu primeiro retorno para revisão da prótese auditiva é agendado para 30 dias após o início do uso. Assim, se opta por orientá-los a fazer o uso da prótese auditiva inicialmente em ambiente caseiro.

Um aspecto que também deve ser pontuado é o medo de roubos e furtos apresentado pelos sujeitos, especialmente pelos idosos, o que os impede de utilizar a prótese auditiva em locais públicos. Bavaresco ${ }^{(25)}$ realizou um estudo sobre a expectativa de idosos sobre o uso de prótese auditiva, no mesmo local onde esta pesquisa foi realizada. Muitos entrevistados relataram que não utilizariam o dispositivo em local público devido ao medo de assaltos.

Pode-se considerar também que os indivíduos, que compuseram a amostra, provavelmente apresentavam deficiência auditiva progressiva e que a capacidade de compreensão de fala já poderia estar bastante afetada. Em função disto, estão sendo realizados novos trabalhos, tentando correlacionar a melhora na qualidade de vida com a capacidade de discriminação de fala apresentada pelo sujeito. Talvez, a melhora nos relacionamentos sociais esteja diretamente relacionada com a capacidade de compreensão.

O domínio meio ambiente também não apresentou dife- rença estatisticamente significante. Isto era esperado, uma vez que as questões deste domínio abordam segurança física; ambiente no lar; recursos financeiros; cuidados de saúde e social (disponibilidade e qualidade); habilidades; oportunidades de informação, recreação e lazer; ambiente físico (poluição, ruído, trânsito, clima) e transporte ${ }^{(16)}$. Assim, não se esperava que houvesse modificação neste domínio com o uso de prótese auditiva, pois são itens que dependem diretamente de políticas públicas para o cidadão.

Os escores pré e pós-protetização, segundo a variável sexo, evidenciaram que, tanto nos indivíduos do sexo feminino quanto nos indivíduos do sexo masculino, foram observadas diferenças estatisticamente significantes no domínio psicológico e nos valores totais. Assim como observado nos resultados do grupo, apesar de não haver diferenças significantes nos demais domínios, houve melhora nos resultados, quando comparados os valores pré e pós-protetização, exceto no domínio social nos sujeitos do gênero masculino.

Este fato pode ser explicado pela maior resistência dos homens ao uso de prótese auditiva, especialmente no âmbito social, o que já foi constatado por outros autores ${ }^{(12,25)}$. Estudos demonstram que os homens apresentam mais resistência, tanto durante a fase de diagnóstico (normalmente são as esposas ou demais membros da família que os levam para avaliação auditiva) quanto ao uso de prótese auditiva. Além disso, as mulheres, em sua maioria, realizam atividades diárias que exigem mais funções comunicativas do que os homens. Muitas vezes, estes optam por utilizar a prótese auditiva somente em situações específicas (ver televisão), o que interfere negativamente no processo de adaptação ${ }^{(25)}$.

Assim, os resultados demonstram que, após o uso da prótese auditiva, há melhora da qualidade de vida como um todo e especialmente no domínio psicológico, em ambos os sexos ${ }^{(12,26)}$, evidenciando a importância do uso de prótese auditiva e do encaminhamento dos usuários para programas de adaptação e treinamento de estratégias de comunicação ${ }^{(27-30)}$. Além disso, é necessário que sejam criados ou implementados programas que visem a reintegração do indivíduo à sociedade, especialmente os idosos. Uma alternativa seria o encaminhamento dos indivíduos a grupos de convivência, visando à melhora dos relacionamentos sociais.

\section{CONCLUSÃO}

A análise dos dados demonstra que houve melhora na qualidade de vida dos indivíduos avaliados após a adaptação da prótese auditiva. A variável sexo não influenciou nos resultados.

Melhorar a qualidade de vida desta parcela da população, que tende a crescer nos próximos anos, é de grande valia social. Assim, a realização de avaliações auditivas e os encaminhamentos para seleção e adaptação de prótese auditiva devem fazer parte da rotina dos profissionais das mais variadas áreas que trabalham com adultos e idosos. 


\begin{abstract}
Purpose: To verify whether adaptation of hearing aids contributes to the improvement of quality of life in adults and elderly people and if the variable gender influences the results. Methods: The sample consisted of 20 individuals, nine women and 11 men, with ages between 45 and 81 years old. The instrument used for data collection was the WHOQOL-bref, which was applied before the hearing aids adaptation and after a one-month use period. Results: Improvement in quality of life was verified comparing pre and post adaptation periods. This improvement was significant in the psychological domain, as well as in the global score results. Gender, as a variable, did not affect the results in the studied group. Conclusion: The adaptation of hearing aids allowed the improvement in the quality of life of the assessed individuals, regardless of gender.
\end{abstract}

Keywords: Quality of life; Hearing aids; Hearing loss; Ageing; Elderly; Adult; Questionnaires

\section{REFERÊNCIAS}

1. Marques ACO, Koslowski L, Marques JM. Reabilitação no idoso. Rev Bras Otorrinolaringol. 2004;70(1):806-11.

2. Veras RP, Caldas CP. Promovendo a saúde e a cidadania do idoso: o movimento das universidades da terceira idade. Ciênc Saúde Coletiva. 2004;9(2):423-32.

3. Veras RP. Em busca de uma assistência adequada à saúde do idoso: revisão da literatura e aplicação de um instrumento de detecção precoce e de previsibilidade de agravos. Cad Saúde Pública. 2003;19(3):70515 .

4. Cook JA, Hawkins DB. Hearing loss and hearing aids treatment options. Mayo Clin Proc. 2006;81(2):234-7.

5. Cerqueira ATAR, Oliveira NIL. Programa de apoio a cuidadores: uma ação terapêutica e preventiva na atenção à saúde dos idosos. Psicol USP. 2002;13(1):133-50.

6. Kochar A, Hildebrand MS, Smith RJ. Clinical aspects of hereditary hearing loss. Genet Med. 2007;9(7):393-408.

7. Mansur LL, Viude A. Aspectos fonoaudiológicos do envelhecimento. In: Netto MP. Gerontologia: A velhice e envelhecimento em visão globalizada. São Paulo: Atheneu, 2002. p.284-96.

8. Fellinger J, Holzinger D, Gerich J, Goldberg D. Mental distress and quality of life in the hard of hearing. Acta Psychiatr Scand. 2007;115(3):243-5.

9. Jerger S, Jerger J. Alterações auditivas: um manual para avaliação clínica. São Paulo: Atheneu, 1989.

10. Russo ICP, Almeida K. Considerações sobre a seleção e adaptação de próteses auditivas para o deficiente auditivo idoso. In: Almeida K, Iorio MCM, organizadores. Próteses auditivas: fundamentos teóricos e aplicações clínicas. São Paulo: Lovise, 1996. p. 177-90.

11. Magni C, Freiberger F, Tonn K. Avaliação do grau de satisfação entre os usuários de amplificação de tecnologia analógica e digital. Rev Bras Otorrinolaringol. 2005;71(5):650-7.

12. Teixeira AR. O Uso de prótese auditiva na melhoria da qualidade de vida de idosos: um estudo comparativo entre usuários e não usuários [tese]. Porto Alegre: Pontifícia Universidade Católica do Rio Grande do Sul; 2005.

13. Teixeira AR, Thedy RB, Jotz GP, Barba, MC. Sintomatologia depressiva em deficientes auditivos adultos e idosos: importância do uso de próteses auditivas. Arq. Int Otorrinolaringol. 2007;11(4):453-8.

14. Fleck MPA, Lela OF, Louzada S, Xavier M, Chachamovich E, Vieira $\mathrm{G}$ et al. Desenvolvimento da versão em português do instrumento de avaliação de qualidade de vida da OMS (WHOQOL-100). Rev Bras Psiq. 1999;21(1):19-28.
15. Neri AL. Qualidade de vida e idade madura. 5a ed. São Paulo: Papirus; 2003.

16. Fleck MPA, Louzada S, Xavier M, Chachamovich E, Vieira G, Santos $\mathrm{V}$ et al. Aplicação da versão em português do instrumento abreviado de avaliação da qualidade de vida "WHOQOL-bref". Rev Saúde Pública. 2000;34(2):178-83.

17. Fleck MPA, Chachamovich E, Trentini C. Projeto WHOQOL-OLD: Método e resultados de grupos focais no Brasil. Rev Saúde Pública. 2003;37(6):793-99.

18. Organização Mundial da Saúde. WHO/PDH/97.3_Geneva: WHO;1997

19. Amaral LCG, Sena APRC. Perfil audiológico dos pacientes atendidos no Núcleo de Atenção Médica Integrada da Universidade de Fortaleza. Fono Atual. 2004;7(27):58-64.

20. Guarinello AC, Cruz MCM. O perfil dos idosos protetizados na clínica de fonoaudiologia da Universidade Tuiuti do Paraná. Fono Atual. 2006;8(35):59-64.

21. Ballantyne J, Martin MC, Martin A. Surdez. 5a ed. Porto Alegre: Artes Médicas, 1995.

22. Veras RP, Mattos LC. Audiology and Aging: literature review and current horizons. Rev Bras Otorrinonaringol. 2007;73(1):128-34.

23. Mulrow CD, Tuley MR, Aguilar C. Sustained Benefits of Hearing Aids. J Speech Hear Res. 1992;35:1402-5.

24. Theddy RB. Sintomatologia depressiva em adultos e idosos: efeitos do uso de próteses auditivas [monografia]. Canoas: Universidade Luterana do Brasil; 2007.

25. Bavaresco A. Estudo da expectativa de idosos quanto ao uso de prótese auditiva [monografia]. Canoas: Universidade Luterana do Brasil; 2006.

26. Mulrow CD, Aguillar C, Endicott JE, Tuley MR, Velez R, Charlip WS et al. Quality-of-life changes and hearing impairment. A randomized trial. Ann Intern Med. 1990;113(3):188-94.

27. Abrams H, Chisolm TH, McArdle R. A cost-utility analisys of adult group audiologic rehabilitation: are the benefits worth the cost? J Rehabil Res Dev. 2002;39(5):549-58.

28. Vuorialho A, Karinen P, Sorry M. Counselling of hearing aid users is highly cost-effective. Eur Arch Otorhinolaryngol. 2006;263(11):98895.

29. Ruchel CV, Carvalho CR, Guarinello AC. A eficiência de um programa de reabilitação audiológica em idosos com presbiacusia e seus familiares. Rev Soc Bras Fonoaudiol. 2007;12(2):95-98.

30. Boothroyd A. Adult Aural rehabilitation: what is it and does it work? Trends Amplif. 2007;11(2):63-71. 